\section{Formalin-induced severe colonic necrosis}
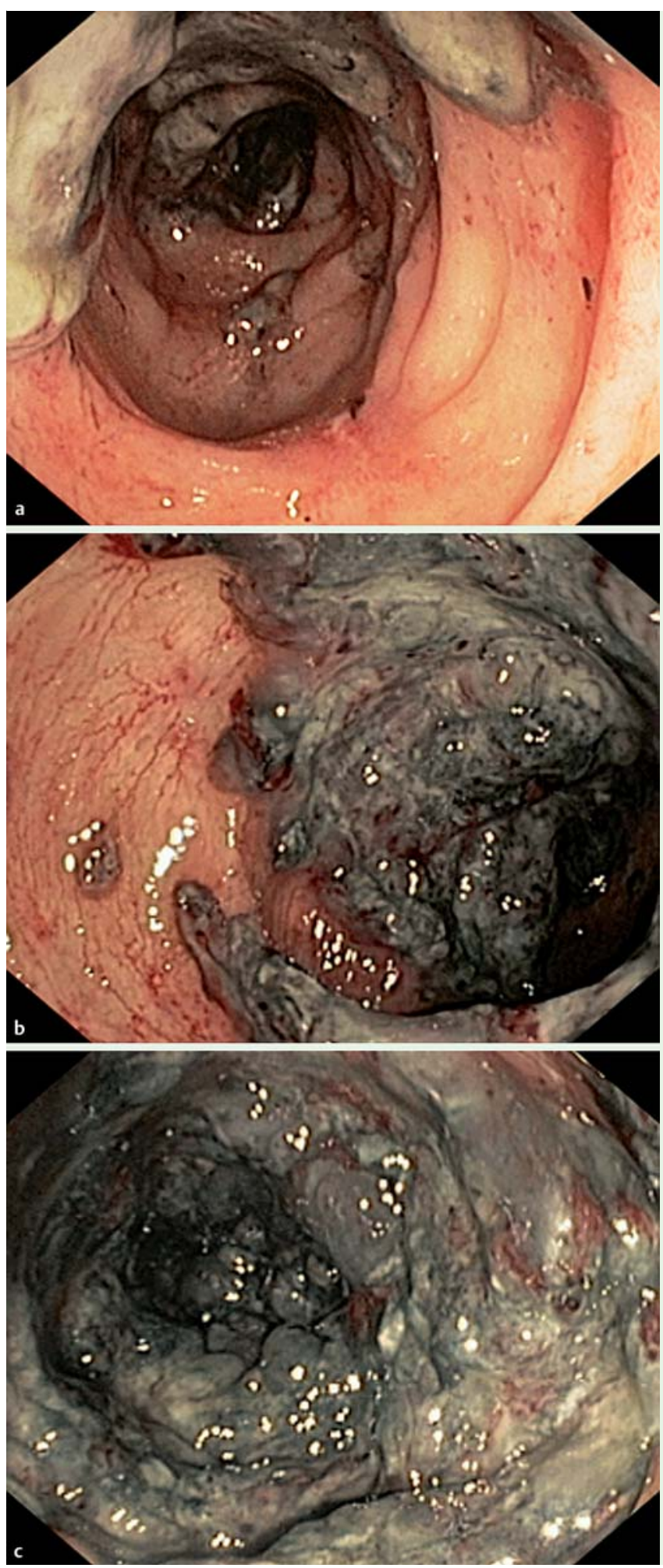

Fig. 1 a Clear demarcation between the normal mucosa and necrosis at descending colon in a 68-year-old woman with diabetes mellitus, end-stage renal disease, and recurring hematochezia. b Note the gray exudate on top of the dark necrotic tissue. c Circumferential mucosal necrosis in the transverse colon.
A 68-year-old woman with diabetes mellitus and end-stage renal disease developed recurring hematochezia after an endoscopic diathermy for diffuse angiodysplasia of the proximal colon. During the second colonoscopy, $100 \mathrm{~mL}$ of $1 \%$ formalin was flushed through to ablate the angiodysplasia. After 2 days, the patient had another episode of hematochezia and was referred to our hospital. She was afebrile and denied abdominal pain. Complete blood count showed moderate anemia with mild leukocytosis. A third colonoscopy showed areas of well-demarcated, extensive necrosis starting at descending colon, and the scope was withdrawn without proximal colon examination ( Fig. 1). Computed tomography (CT) of the abdomen showed arterial enhancing and thickening of the bowel wall, extending from the descending colon to the cecum. No pneumatosis intestinalis was detected ( $\bullet$ Fig.2). Patient management included nil by mouth, intravenous fluids, and broad-spectrum antibiotics. On the fourth day after the formalin treatment, a follow-up CT scan demonstrated further extension of the colonic injury and the possibility of colonic perforation was considered. Consequently, the patient underwent total colectomy with ileorectal anastomosis, with no postoperative complications. Histopathological examination revealed mucosal sloughing, and a large area of hemorrhagic infarction of the mucosa and submucosa, without evidence of perforation ( Fig.3).

Formalin, either by direct administration or using a formalin-soaked gauze, is used for the treatment of radiation-induced hemorrhagic proctitis. This technique has a $39 \%-100 \%$ rate of bleeding cessation [1]. Formalin has a chemical ablative effect on the mucosa but not on submucosal vessels [2], and the reported rates of complications including ulceration and stricture of rectum are $12 \%$ and $18 \%$, respectively [1]. Precautions to be taken while using formalin are: the concentration of formalin should not exceed $4 \%$, and saline flushing is required to clear out the formalin after administration [1 - 3]. Usually formalin is rapidly evacuated from the rectum and proximal colonic injury may develop following delayed evacuation. Therefore, we advise against formalin application in the gastrointestinal tract beyond the rectum.

Endoscopy_UCTN_Code_CPL_1AJ_2AZ 


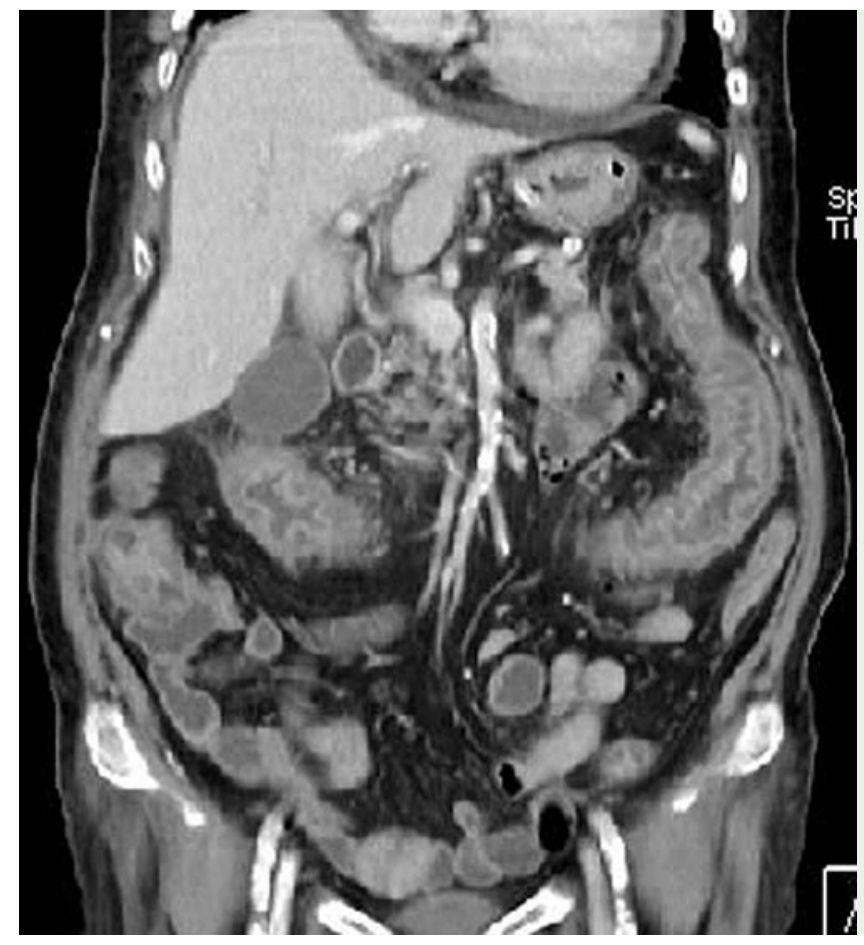

Fig. 2 Computed tomography (CT) coronal view demonstrating arterial enhancing and thickening of the bowel , wall with pericolonic fat stranding, extending from the descending colon to the cecum.

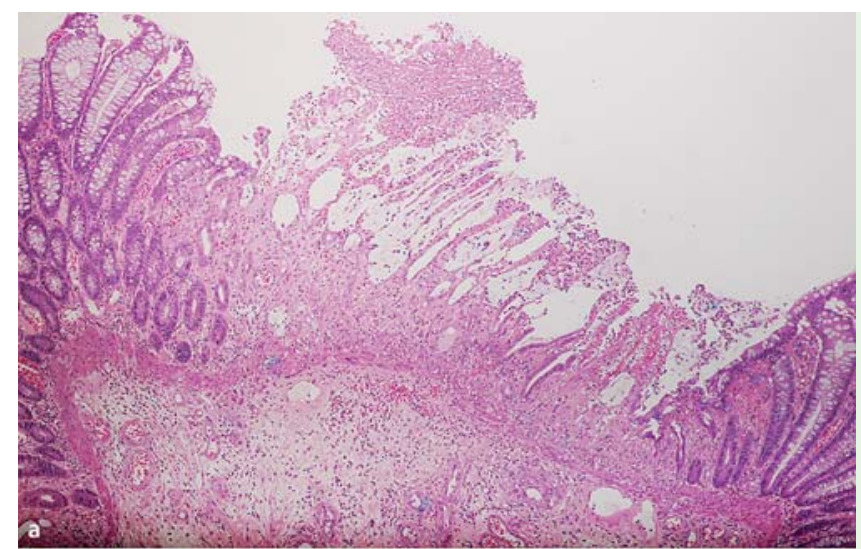

Fig. 3 a Histopathologic section showing infarction of the mucosa and submucosa with areas of ulceration covered by fibrinopurulent material. b Submucosa exhibiting markedly edematous epithelium with congested vessels.

\section{S. Sallapant ${ }^{1}$, P. Angsuwatcharakon ${ }^{2}$, C. Thiptanakit ${ }^{3}$, N. Wisedopas ${ }^{4}$, \\ R. Rerknimitr ${ }^{1}$}

${ }^{1}$ Division of Gastroenterology, Department of Medicine, Faculty of Medicine, Chulalongkorn University, Bangkok, Thailand

${ }^{2}$ Department of Anatomy, Faculty of Medicine, Chulalongkorn University, Bangkok, Thailand

${ }^{3}$ Division of Colorectal surgery, Department of Surgery, Faculty of Medicine, Chulalongkorn University, Bangkok, Thailand

${ }^{4}$ Department of Pathology, Faculty of Medicine, Chulalongkorn University, Bangkok, Thailand

\section{References}

1 de Parades V, Etienney I, Bauer P et al. Formalin application in the treatment of chronic radiation-induced hemorrhagic proctitis an effective but not risk-free procedure: a prospective study of 33 patients. Dis Colon Rectum 2005; 48: 1535 - 1541

2 Myers JA, Hollinger EF, Mall JW et al. Mechanical, histologic, and biochemical effects of canine rectal formalin instillation. Dis Colon Rectum 1998; 41: 153-158

3 Munoz-Navas M, Garcia-Villareal L. Caustic colitis due to formalin enema. Gastrointest Endosc 1992; 38: 521-522

\section{Bibliography}

Dol http://dx.doi.org/

10.1055/s-0033-1344772

Endoscopy 2013; 45: E363-E364

(c) Georg Thieme Verlag KG

Stuttgart · New York

ISSN 0013-726X

\section{Corresponding author \\ R. Rerknimitr}

Division of Gastroenterology

Department of Internal Medicine

Faculty of Medicine

Chulalongkorn University

Bangkok 10310

Thailand

Fax: +66-2-2527839

ERCP@live.com 